\title{
Antidiabetic Drugs in Alzheimer's Disease and Mild Cognitive Impairment: A Systematic Review
}

\author{
Mario Muñoz-Jiménez ${ }^{a} \quad$ Alí Zaarkti $^{a} \quad J u a n$ Antonio García-Arnésa, b \\ Natalia García-Casares ${ }^{\text {a, c, d }}$ \\ aDepartment of Medicine, Faculty of Medicine, University of Málaga, Málaga, Spain; b Department of Pharmacology, \\ Faculty of Medicine, University of Málaga, Málaga, Spain; 'Centro de Investigaciones, Médico-Sanitarias (C.I.M.ES),

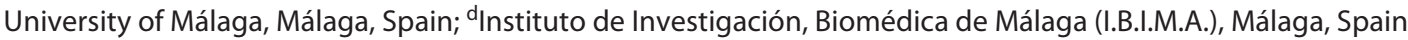

\section{Keywords}

Alzheimer's disease · Mild cognitive impairment ·

Antidiabetic drugs · Cognitive functioning

\begin{abstract}
Introduction: Considering that Alzheimer's disease (AD) and diabetes mellitus share pathophysiological features and AD remains with no cure, antidiabetic drugs like intranasal insulin, glitazones, metformin, and liraglutide are being tested as a potential treatment. Objective: The aim of this systematic review was to assess the efficacy of antidiabetic drugs in patients with $A D$, mild cognitive impairment $(\mathrm{MCl})$, or subjective cognitive complaints (SCCs). Cognition was studied as the primary outcome and modulation of AD biomarkers, and imaging was also assessed as a secondary outcome. Methods: We conducted a search in the electronic databases PubMed/MEDLINE, EMBASE, and Scopus seeking clinical trials evaluating the effect on cognition of antidiabetic drugs in patients with AD, MCl, or SCCs. Results: A total of 23 articles were found eligible. Intranasal regular insulin improved verbal memory in most studies, especially in apoE4- patients, but results in other cognitive domains were unclear. Detemir improved cognition after 2 months of treatment,
\end{abstract}

but it did not after 4 months. Pioglitazone improved cognition in diabetic patients with $\mathrm{AD}$ or $\mathrm{MCl}$ in 3 clinical trials, but it is controversial as 2 other studies did not show effect. Metformin and liraglutide showed promising results, but further research is needed as just 2 clinical trials involved each of these drugs. Almost all drugs tested were shown to modulate AD biomarkers and imaging. Conclusions: Intranasal insulin, pioglitazone, metformin, and liraglutide are promising drugs that could be useful in the treatment of AD. However, many questions remain to be answered in future studies, so no particular antidiabetic drug can currently be recommended to treat AD.

๑) 2020 S. Karger AG, Basel

\section{Introduction}

Alzheimer's disease (AD) is the most common cause of dementia, corresponding to about $60 \%$ of cases [1], reaching a prevalence of 40.2 per 1,000 among individuals older than 60 years [2]. It results in disability and dependency and, therefore, serious consequences for family and society [1]. In 2011, the National Institute on Ageing (NIA) at National Institutes of Health (NIH) and the Alzheimer's As-

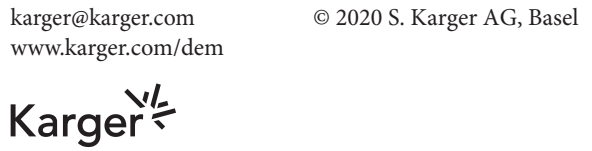

Natalia García-Casares

Department of Medicine, Faculty of Medicine, University of Málaga Boulevard Louis Pasteur, 32 ES-29010 Málaga (Spain)

Nagcasares@uma.es 
sociation published revised guidelines (NIA-AA) and created 3 separate diagnostic recommendations: mild cognitive impairment (MCI) and dementia (for symptomatic or "clinical" stages of $\mathrm{AD}$ ) and preclinical $\mathrm{AD}$ (for a stage of $\mathrm{AD}$ in individuals without overt symptoms) [3]. Most studies today further differentiate between amnestic MCI (aMCI) and nonamnestic MCI depending on whether or not memory is impaired [4]. MCI patients are at increased risk of progression to $\mathrm{AD}$ or dementia, and $\mathrm{aMCI}$ is seen to be highly associated with progression to AD. However, not all subjects with $\mathrm{MCI}$ will develop $\mathrm{AD}$ or dementia (although they are still at greater risk than cognitively normal subjects), and some will remain stable or even return to normal cognition [4]. One of the earliest symptoms of $\mathrm{AD}$ is subjective cognitive complaints (SCCs), typically expressed as memory concerns. Patients with SCCs are at higher risk of developing $\mathrm{MCI}$ and dementia due to $\mathrm{AD}$, but most of them do not experience objective cognitive decline [5]. Further research is needed to ascertain which parameters are related to progression, and preventive clinical trials in this population are already under way [5].

In 2018, the NIA-AA workgroup proposed that Alzheimer's disease should be defined as a pathophysiological construct toward a biological definition of Alzheimer's disease. As in other diseases, such as diabetes, biomarkers would alone define the presence of the disease in a living person regardless of their symptoms [6].

Despite the great amount of investigation carried out, $\mathrm{AD}$ remains without a cure and the only drugs approved for its treatment (the last in 2002) simply ameliorate symptoms and have just a modest effect at slowing cognitive and functional decline $[3,7]$. New disease-modifying drugs with stronger effects are needed, but research has been centered on the "amyloid cascade hypothesis" for 20 years, with all attempts to develop new drugs failing [8]. In this context, there is growing interest in a hypothesis linking diabetes mellitus (DM) and AD. Insulin has important functions in the brain affecting synapsis, neuronal and glial metabolism and trophism, and the neuroinflammatory response. Consequently, it regulates memory and other cognitive and emotional functions [9]. Furthermore, it is known that type 2 diabetes is a risk factor for cognitive impairment and dementia, thus the benefits of antidiabetics on cognition in patients with diabetes has been a focus of interest $[10,11]$. In this way, it has been recently reported that long-term treatment with the glucagon-like peptide-1 (GLP-1) receptor agonist dulaglutide might reduce cognitive impairment in type 2 diabetes patients in the exploratory analysis within the Researching Cardiovascular Events With a Weekly Incretin in Di- abetes (REWIND) trial [12]. Likewise, antidiabetic drugs represent a promising treatment for neurodegenerative diseases such as $\mathrm{AD}[13,14]$. Brain insulin resistance and impaired glucose metabolism are features of $\operatorname{AD}[9,14$, $15]$. They precede the clinical expression of $A D$ and correlate with the severity of cognitive impairment $[16,17]$. Moreover, insulin resistance boosts other hallmarks of $\mathrm{AD}$, such as $\mathrm{A} \beta$ deposition and tau phosphorylation [18], though the pathophysiological interactions are complex and not well understood, and it is not clear which pathological feature really initiates the process [9].

These findings opened the door to research on antidiabetic drugs for AD. First studies with insulin involved the intravenous route, but it is impossible to use in clinical practice as it would provoke hypoglycemia and systemic insulin resistance. The intranasal route was developed in order to solve this problem. It raises insulin levels in the central nervous system without modifying plasma levels, so systemic adverse events are avoided. Agents like metformin, glitazones, and GLP-1 agonists are also interesting options due to their insulin-sensitizing effect and other direct actions independent of insulin-signaling mechanisms that could be beneficial for cognitive impairment.

Several preclinical and clinical studies involving antidiabetic agents have obtained promising results, modifying the pathological and clinical progression of $\mathrm{AD}$ and improving cognition [19]. In order to examine the potential use of antidiabetic agents for the treatment of $\mathrm{AD}$, we reviewed randomized clinical trials (RCTs) that assessed the efficacy of antidiabetic agents in patients with probable AD, MCI, or SCCs. We used cognitive function as the primary outcome and $\mathrm{AD}$ biomarkers and imaging as secondary outcomes.

\section{Methods}

We followed the Preferred Reporting Items for Systematic reviews and Meta-Analyses (PRISMA) guidelines [20].

\section{Eligibility Criteria}

We only included studies meeting the following eligibility criteria: (1) RCTs enrolling humans. (2) Patients of any age with AD, MCI, or SCCs. (3) At least 1 group of patients treated with 1 or more antidiabetic drugs, excluding intravenous insulin. (4) Cognitive function assessed before and after the intervention. (5) Articles written in English or Spanish and published or accepted for publication.

\section{Information Sources}

Studies were found by searching the electronic databases and reviewing reference lists of included articles and related reviews. PubMed/MEDLINE, EMBASE, and Scopus were searched on June 2020. 


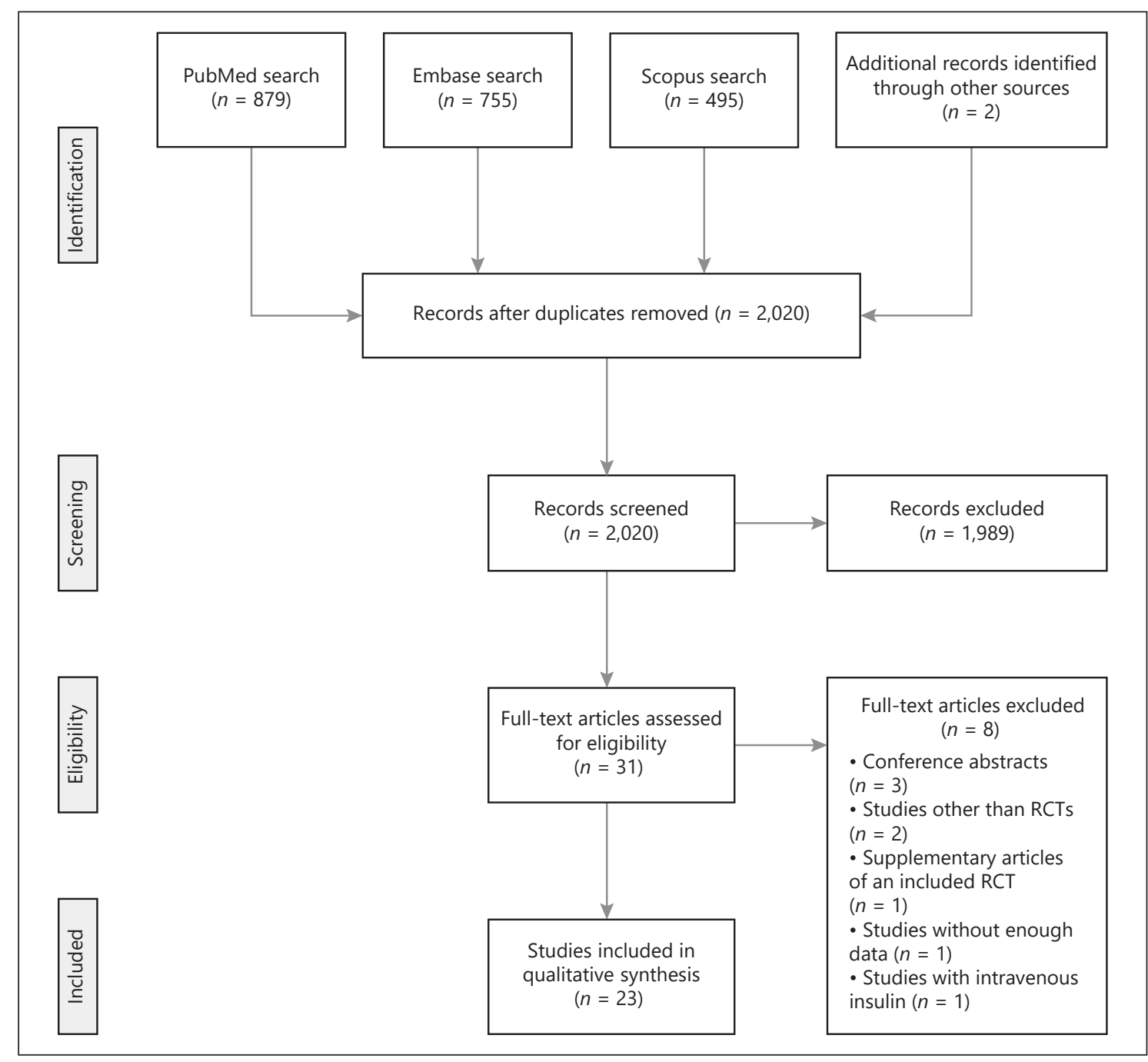

Fig. 1. Study selection flow diagram.

Search

Terms related to antidiabetic drugs, $\mathrm{AD}$, and clinical trial were combined in the search query using the Boolean operators OR and AND: "pioglitazone," "biguanide," "insulin," "Alzheimer," "cognitive impairment," "memory," "cognition," "clinical trial," "randomized controlled trial," and "double blind."

\section{Study Selection}

Two reviewers (A.Z. and M.M.J.) independently conducted the evaluation of the studies. Disagreements were resolved by a third author (N.G.C.) and consensus. The study selection consisted of 2 phases: a screening phase, assessing the title and abstract, and a full-text evaluation phase to determine eligibility.

\section{Data Collection Process}

We designed a data extraction form in Excel and pilot-tested it on 8 random studies in order to refine the form. The data were then collected by 2 reviewers (A.Z. and M.M.J.) independently and dis- agreements were resolved by a third author (N.G.C.) and consensus.

\section{Data Items}

The data extraction form contained the following items: first author, year of publication, title, study design, stratified randomization (whether this was done or not and variables for stratification), number of participants randomized, characteristics of the participants (type of cognitive impairment and number of participants of each type, whether diabetic patients were included or not, and other baseline characteristics), AD, MCI, and DM criteria, antidiabetic drug administered, other anti-Alzheimer or antidiabetic drugs permitted, intervention and control groups and their number of patients, duration of intervention, study objectives, outcome variables (cognitive and functional tests, AD biomarkers, imaging, and adverse effects), and main findings. 


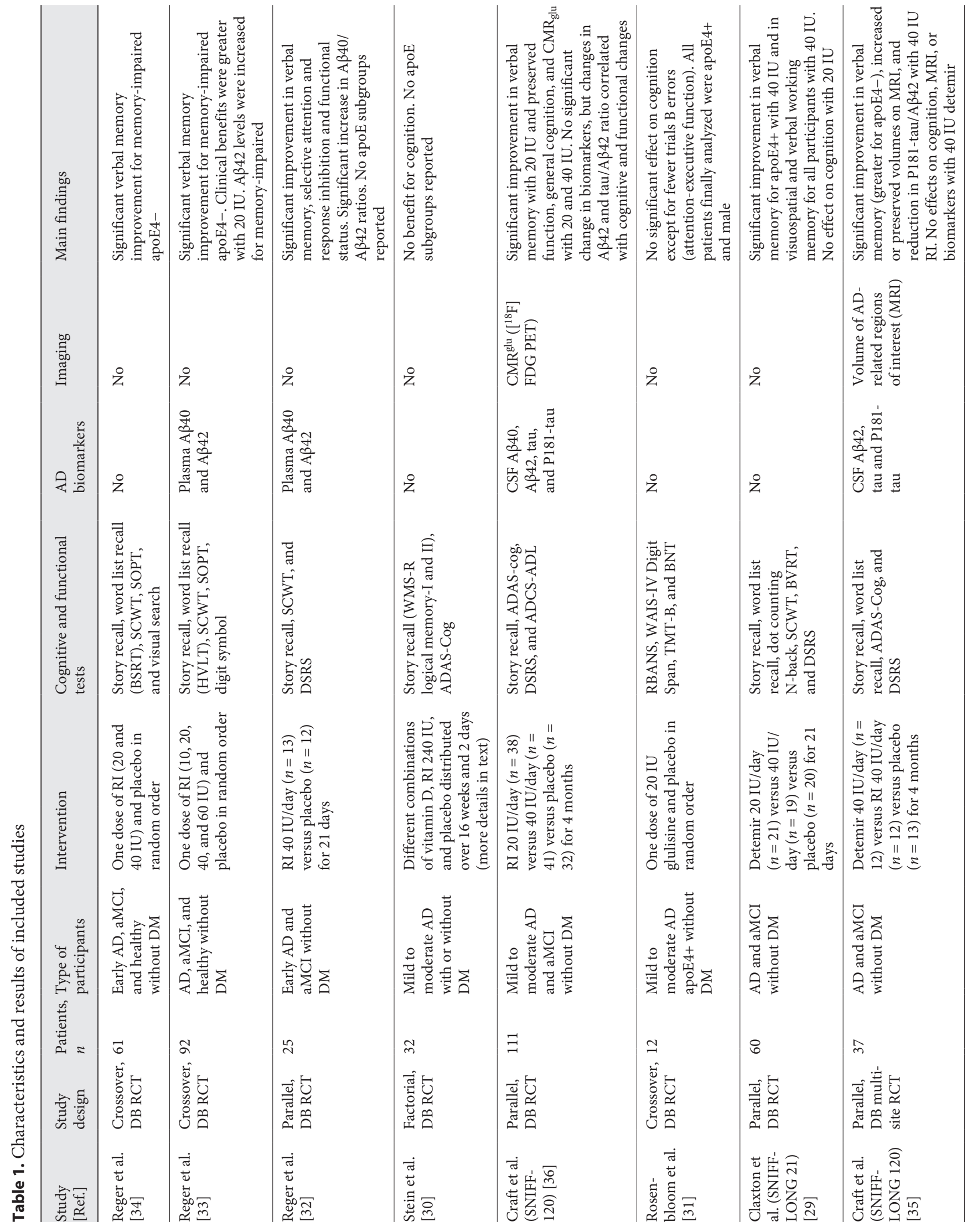




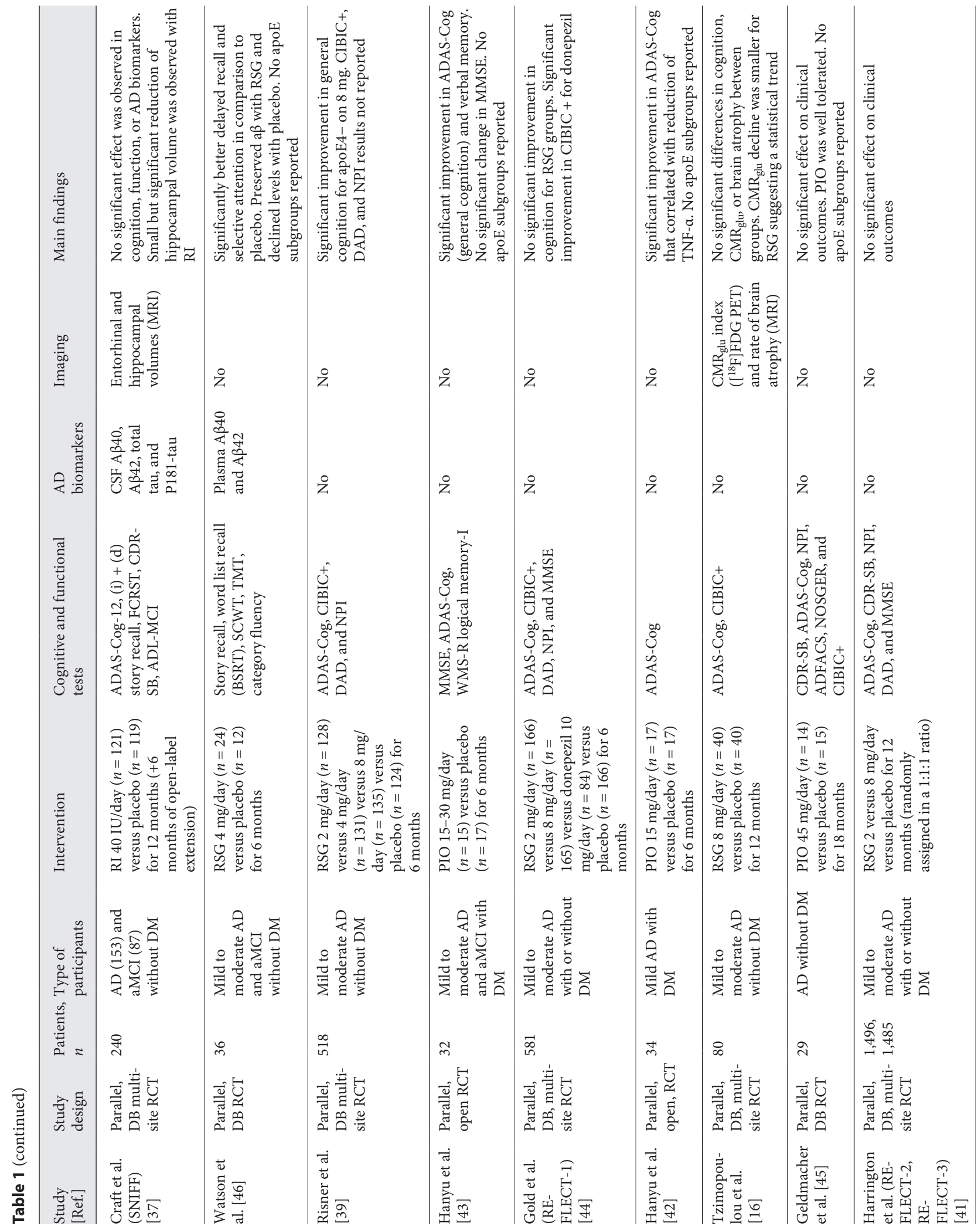




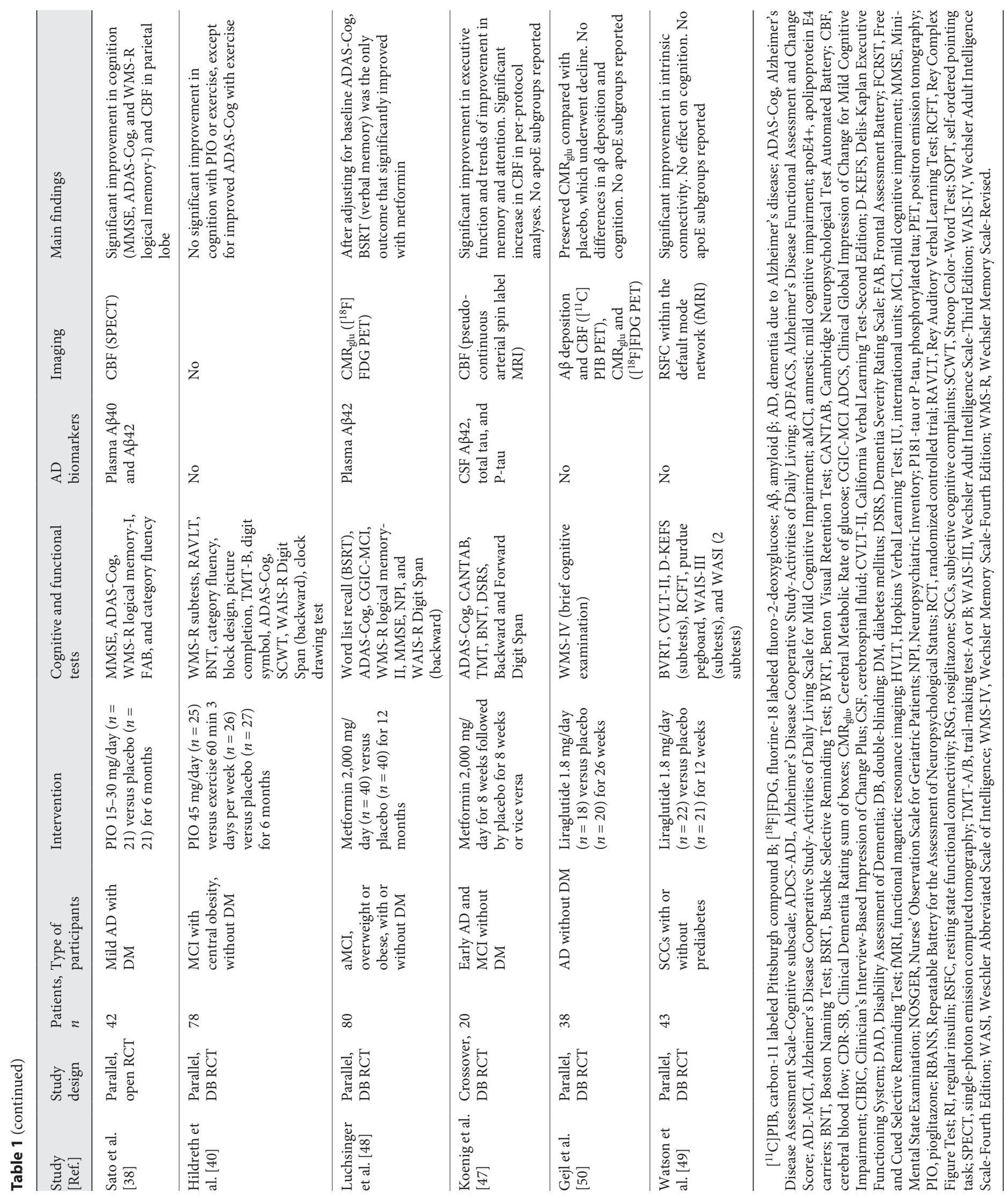




\section{Results}

\section{Study Selection}

Of the 2,131 records identified, 2 were obtained reviewing the reference lists of included articles and related reviews and the remainder through database search. After removing duplicates, 2,020 records were screened, leaving 31 full-text articles that were assessed for eligibility. Three articles were excluded because they were conference abstracts [21-23]. Two articles were excluded as they were not RCTs [24, 25]. One article was excluded because it was a supplementary analysis [26]. Another was excluded as it did not have enough data about cognitive outcomes [27]. One more article was excluded because the intervention drug was intravenous insulin [28]. Figure 1 contains the flow diagram of study selection.

\section{Study Characteristics}

Table 1 contains the 23 articles that proved eligible for inclusion and their characteristics and results. Intranasal insulin was used in 9 studies, involving $n=670$ patients [29-37], oral glitazones (rosiglitazone or pioglitazone) were used in 10 studies, involving 4,411 patients [16, 3846], oral metformin was used in 2 studies, involving 100 patients $[47,48]$, and subcutaneous liraglutide in another 2 studies, involving 81 patients [49, 50]. Most studies included patients with $\mathrm{AD}$ or MCI, but Watson et al. [49] recruited patients with SCCs and Reger et al. $[33,34]$ also included healthy participants. Various studies included only patients with known insulin resistance (DM or obesity) $[36,40,42,43,48]$. The study by Rosenbloom et al. [31] was the only study which just included subjects with apolipoprotein E4 (apoE4).

Stein et al. [31] used a factorial design. All the participants received 1,000 IU of vitamin D2 daily for the full duration of the trial. After 8 weeks, the participants were randomized to $36,000 \mathrm{IU} /$ day of vitamin $\mathrm{D} 2$ or placebo for 8 weeks. At the end of this period, the participants were randomized to $240 \mathrm{IU} /$ day of intranasal insulin or placebo for 2 days [30]. Harrington et al. [41] included 2 clinical trials (REFLECT-2 and REFLECT-3). In both trials, acetylcholinesterase inhibitors were mandatory in all participants, using just donepezil in REFLECT-2 and any acetylcholinesterase inhibitor in REFLECT-3 [41]. The trial of Hildreth et al. [40] was the only one that used exercise (walking on a treadmill) as an intervention. Three pioglitazone clinical trials were not blinded (open clinical trials) $[38,42,43]$.

Antidiabetic Drugs in Alzheimer's Disease
Results of the Studies

Intranasal Insulin

Regular insulin improved cognition in 5 clinical trials [32-36]. In crossover trials with 1 dose of regular insulin per intervention, regular insulin just improved verbal declarative memory in memory-impaired subjects without apoE4 $[33,34]$, but in a 21-day clinical trial, it enhanced verbal declarative memory, selective attention, and response inhibition in all treated subjects and functional status in participants with more severe cognitive impairment [32]. Two trials studied regular insulin for 4 months. Both obtained an improvement in verbal declarative memory $[35,36]$. However, Craft et al. $[35,36]$ did not corroborate the preservation of general cognition and functional status obtained in Craft et al. $[35,36]$. The doses that proved to obtain better results on cognition were 20 and 40 IU per day [32-36]. ApoE4+ participants did not benefit from regular insulin as apoE4- patients did, achieving worse results [35] and showing no cognitive facilitation or even reduced performance [33, 34]. Only 1 study reported apoE4 modulation but did not demonstrate any differences [36]. Recently, results of the SNIFF study have been reported. Craft et al. [37] tested 240 participants for a blinded period of 12 months and an additional open-label period of 6 months. No cognitive or functional benefits were observed with 40 IU intranasal insulin treatment over a 12 -month period among the primary intention-to-treat cohort.

Stein et al. [30] found no effect on cognition with regular insulin. In a 21-day clinical trial, 40 IU detemir (a long-acting insulin) improved verbal declarative memory for apoE4+ patients and visuospatial and verbal working memory for all participants [29]. However, it failed to modulate cognition in another trial that lasted 4 months. Detemir-treated apoE4+ participants showed greater preservation of memory than placebo at month 2 , but this effect was not observed at month 4 [35].

Rosenbloom et al. [31] explored the effect of glulisine (a rapid-acting insulin) on cognition in apoE4+ patients but found no benefit. They only observed better results than placebo in one attention and executive function test, but this should be interpreted with caution as no other significant differences were found in other similar tests [31].

Regular insulin modified biomarker levels in 3 clinical trials $[32,33,35]$. However, it failed to do so in another trial, but changes in biomarkers correlated with cognitive and functional changes [36]. Results were conflicting. The effect of detemir on biomarkers was explored by Craft et al. [35], but no change was observed. Regular insulin preserved cerebral metabolic rate of glucose

Dement Geriatr Cogn Disord 2020;49:423-434 DOI: $10.1159 / 000510677$ 
$\left(\mathrm{CMR}_{\mathrm{glu}}\right)$ in one clinical trial [36] and increased or preserved the volume of $\mathrm{AD}$-related regions of interest in another [33]. Small but significant reductions in hippocampal volume were found for the regular insulin arm in the SNIFF study [37]. No serious adverse effects were observed, and most of these were nasal symptoms [29-36].

\section{Glitazones}

Rosiglitazone improved cognition in the first two 6 -month clinical trials $[39,46]$ but failed to have an effect in the next studies performed (one lasted 6 months and the other three 12 months) [16, 41, 44]. All studies reported results for apoE subgroups [16, 39, 41, 44] except for the first [46]. Only Risner et al. [39] found differences between apoE4+ and apoE4- patients. While apoE4- patients improved general cognition with $8 \mathrm{mg}$, apoE4+ patients showed no improvement (and even worsened) with $2 \mathrm{mg}$ [39].

Pioglitazone improved cognition (verbal declarative memory and general cognition) in 3 clinical trials with diabetic patients at $15-30 \mathrm{mg} /$ day $[38,42,43]$ but failed to have an effect in 2 clinical trials at $45 \mathrm{mg} /$ day in nondiabetic patients $[40,45]$ (with central obesity in one of them [40]). Two studies analyzed results according to the apoE4 status [38, 40]. Sato et al. [38] found no correlation. Hildreth et al. [40] observed significantly worse visuospatial scores with pioglitazone than placebo in apoE4- patients. Memory, language, and executive domains showed better results for pioglitazone than placebo in apoE4- patients, but statistical significance was not reached. These results should be interpreted with caution due to the small sample size [40].

Plasma $A \beta 40$ and $A \beta 42$ were measured in 1 rosiglitazone trial [47] and in 1 pioglitazone trial [38]. Rosiglitazone preserved both $\mathrm{A} \beta$ levels compared with placebo, where the levels declined [46]. Pioglitazone did not modify A $\beta$ levels [38]. Tzimopoulou et al. [16] studied the $\mathrm{CMR}_{\text {glu }}$ index and rate of brain atrophy response to rosiglitazone, just obtaining a statistical trend suggesting a smaller decline in the $\mathrm{CMR}_{\text {glu }}$ index. Sato et al. [38] showed that pioglitazone enhances cerebral blood flow $(\mathrm{CBF})$ in the parietal lobe.

\section{Metformin}

Metformin improved cognition in the 2 clinical trials included but did not change $\mathrm{AD}$ biomarkers in either of them $[47,48]$. One study measured $\mathrm{CMR}_{\mathrm{glu}}$ but found no effect on it [48]. The other trial measured CBF and observed a significant increase in per-protocol analyses [47]. Only Luchsinger et al. [48] stratified results by apoE4 sub- groups, finding a better cognitive performance in apoE4participants.

\section{Glp-1 Analogs}

Neither of the 2 studies included showed cognitive improvement $[48,49]$. Liraglutide prevented $\mathrm{CMR}_{\text {glu }}$ decline in 1 clinical trial [50] and enhance intrinsic connectivity within the default mode network in the other [49]. ApoE4 subgroups were not analyzed $[49,50]$.

\section{Discussion}

Despite the known relationship between insulin resistance and $\mathrm{AD}$ and all the clinical trials that have been performed, there is still not enough evidence to recommend any antidiabetic agent as a treatment of dementia, MCI, or SCCs due to AD. Results are promising for almost all reviewed drugs, especially for intranasal regular insulin that has the best and most consistent outcomes for the moment. However, almost all clinical trials are phase 2, so further research is needed.

Insulin sensitizers deserve special attention as their mechanisms of action could be better than insulin ones. They have insulin-independent effects and improve insulin sensitivity [51, 52]. Insulin administration can keep insulin stimulation despite insulin resistance, but it could worsen it in the long term. New routes and formulations should also be explored. Some antidiabetic drugs like glitazones or dipeptidyl peptidase- 4 inhibitors do not cross the blood-brain barrier well [52-55], and this could entail a limitation on their beneficial effect. The intranasal route could enhance brain bioavailability and reduce systemic adverse events [51].

\section{Intranasal Insulin}

Intranasal regular insulin appears to improve verbal declarative memory, especially in apoE4- patients, as observed in almost all clinical trials [32-36]. However, recently Craft et al. [37] did not demonstrate the benefits of intranasal insulin treatment for any outcome during 12 months using an insulin delivery device not previously used in clinical trials of persons with $\mathrm{AD}$ but with excellent rates of adherence $(>90 \%)$. The authors indicated that further investigation using reliable insulin delivery devices verifying the ability to elevate insulin in the central nervous system is needed to assess the therapeutic benefit of intranasal insulin for the treatment of MCI and AD. Additionally, another study did not show improvement combining regular insulin with vitamin $\mathrm{D}$ and using huge dos- 
es of regular insulin, so these special conditions could explain the failure [30]. ApoE4 status seems to be an important modulator of intranasal insulin effects. ApoE4+ patients do not seem to respond or even worsen with insulin administration, as was previously observed in other studies [56]. This could be related to the fact that ApoE4+ patients have metabolic brain abnormalities $[57,58]$ and mitochondrial dysfunction in the posterior cingulate gyrus [59]. On the other hand, apoE4- $\mathrm{AD}$ patients tend to have greater insulin resistance and; therefore, memory facilitation is greater with insulin administration. For the same reason, apoE4- patients need higher insulin doses to improve cognition [56], so maybe the doses used in these clinical trials were too high for apoE4+ patients. Detemir was the only drug that obtained better results in apoE4+ than apoE4- patients [29]. Unfortunately, these results weakened and lost significance with a longer intervention [35]. Different mechanisms as chronic hyperinsulinemia, capacity of binding to albumin, or slower clearance from the central nervous system have been suggested to explain the declining effect of detemir along time [35], which could be the greatest limitation of long-acting insulins.

Results in other cognitive domains are not clear enough to draw conclusions, as just a few clinical trials explored each of them and results were conflicting. Glulisine did not show an effect on cognition, but clinical trial enrolled a very small sample of apoE4+ patients [31], so glulisine should not be dismissed yet. In fact, it is thought that rapid-acting insulins could be more effective than regular insulin as their pharmacokinetic characteristics are opposed to those of long-acting insulins. Moreover, a study enrolling healthy men obtained better memory improvement with insulin aspart (a rapid-acting insulin) than with regular insulin [60].

The main research queries that remain to be explored with intranasal insulins in larger and longer clinical trials are: (1) effect on cognition excluding verbal declarative memory, of which results are more consistent, (2) efficacy of rapid-acting insulins, and (3) differential effect on apoE4 carriers and non-carriers. Two clinical trials addressed these questions in patients with probable $\mathrm{AD}$ and MCI. The SNIFF-Quick study investigated 40 IU insulin aspart effect in 30 subjects for 12 weeks. It was completed in September 2019 (ClinicalTrials.gov NCT02462161). An ongoing study is testing $40 \mathrm{IU}$ insulin glulisine in 90 subjects for 6 months. It is close to completion (ClinicalTrials.gov NCT02503501).

\section{Glitazones}

The initial results with rosiglitazone were promising $[39,46]$, but later clinical trials with larger numbers of patients and a longer duration achieved very poor results $[16,41,42]$. Moreover, its use as rosiglitazone was withdrawn from the market due to increased cardiovascular risk [61].

Pioglitazone, however, is still a promising candidate. It seems to improve verbal declarative memory, general cognition, and $\mathrm{CBF}$ in mild $\mathrm{AD}$ diabetic patients as was observed in 3 clinical trials and demonstrated the greatest efficacy compared to placebo in network meta-analysis $[38,42,43,62]$. Nevertheless, results are quite controversial since 2 other clinical trials proved no efficacy of pioglitazone $[40,45]$. Differences between studies could explain these results. First-mentioned studies were unblinded and enrolled patients with DM $[38,42,43]$, whereas the other studies were blinded and did not recruit diabetic patients $[40,45]$ (one of them enrolled patients with central obesity as a proxy for insulin resistance instead [40]). Then, results could be due to biases or modulation by diabetic status. Furthermore, samples of all studies were very small $[38,40,42,43,46]$. Both articles not showing modulation of cognition discussed they were underpowered for this reason $[40,45]$, but it was especially true for 1 of them which was focused on safety outcomes [45]. The sample of the other one was similar to clinical trials performed in diabetic patients, even quite bigger [38, 40, 42, 43]. We can conclude that further research is needed. Longer, larger, and blinded clinical trials enrolling patients with and without DM could solve these questions.

The TOMORROW clinical trial started in 2013 aiming to determine if low-dose pioglitazone $(0.8 \mathrm{mg})$ could delay the onset of MCI due to $\mathrm{AD}$ in cognitively normal participants at high risk (ClinicalTrials.gov NCT01931566). Its extension study tested the effect in high-risk participants that developed MCI during the TOMORROW trial (ClinicalTrials.gov NCT02284906). These studies were supported by the idea that a much lower dose than the one used for DM could increase $A \beta$ clearance through the blood-brain barrier [63], but both clinical trials were terminated due to lack of efficacy.

A possible explanation for the limited success of research on glitazones could be brain bioavailability as it has been observed that transport of glitazones into the central nervous system is limited by efflux transporters $[55,56]$. Therefore, concentration in the central nervous system could be too low to exert direct actions in brain cells. A novel nanoformulation for intranasal use has shown to significantly improve the concentration of pioglitazone reaching the brain in rats [64].

Regarding correlation between glitazones effect and apoE4 status, results were controversial too. Five clinical 
trials with rosiglitazone $[16,39,41,45]$ and 2 with pioglitazone $[38,40]$ analyzed it, but just 1 study for each drug showed different results for apoE4- and apoE4+ [39, 40]. A recent meta-analysis addressed this question synthesizing results of 4 rosiglitazone clinical trials $[39,41,45]$ and obtained that, compared to placebo, rosiglitazone significantly improved ADAS-Cog in apoE4- patients and significantly worsened it in apoE4+ participants. Although the number of studies was small, this meta-analysis suggests that apoE4 status affects the efficacy of glitazones [65]. Future clinical trials with pioglitazone should explore this possibility.

\section{Metformin, GLP-1 Analogs, and Other Drugs}

Metformin improved cognition and $\mathrm{CBF}$ in $\mathrm{AD}$ and MCI patients $[48,49]$ and liraglutide preserved $\mathrm{CMR}_{\mathrm{glu}}$ in $\mathrm{AD}$ patients [51] and improved intrinsic connectivity of the default mode network in SCC patients [50]. Liraglutide did not modulate cognition, but both studies were underpowered for cognitive outcomes [50,51]. These results are still too naïve to draw definite conclusions but encourage further research. Accordingly, the MAP study, a 2-year metformin clinical trial, is starting in 2020 (ClinicalTrials.gov NCT04098666) and the ELAD study, another one testing liraglutide is ongoing trial registration (ClinicalTrials.gov NCT01843075) [66]. In addition, current research is extending to other antidiabetic drugs like dapagliflozin which also has an ongoing clinical trial (ClinicalTrials.gov NCT03801642).

\section{Biomarkers and Imaging}

Several clinical trials evidenced that antidiabetics can modulate $\mathrm{AD}$ biomarkers and imaging parameters [32, $33,35,36,46,47,49,50]$. They suggest that antidiabetic drugs could affect pathological changes of the disease [33, $36-38,46,47,49,50]$; however, these studies were mostly exploratory including a small number of patients and, therefore, should be interpreted with caution.

\section{Limitations}

This systematic review has potential limitations. It was difficult to extract firm conclusions because of the great heterogeneity of the studies. The sample size, duration and design of interventions, outcome measures investigated, and analyses performed differed greatly between the studies. An important consideration is that many studies are underpowered to detect a clinically significant difference in cognition. Among the addressed studies, 18 of them had $<100$ patients in total and none over 600 patients. Additionally, some of the studies were of short treatment duration. These facts have an impact on how to interpret the results. Search and selection of studies were conducted carefully, but we cannot guarantee that we found all clinical trials as the search did not aim for unpublished studies.

\section{Conclusion}

The present systematic review analyzes the effect of antidiabetic agents on SCCs, MCI, and dementia due to $\mathrm{AD}$ that has been observed in clinical trials. Intranasal insulin, pioglitazone, metformin, and liraglutide may improve cognition (especially verbal declarative memory) as well as AD biomarkers and imaging parameters. Intranasal regular insulin has the greatest evidence to support its efficacy for the moment, especially in verbal declarative memory. ApoE4 status seems to be an important modulator of response. The results are promising but are insufficient to recommend antidiabetics to treat SCCs, MCI, or dementia due to AD. Further research is necessary in this field. Influence of apoE4 status, DM, stage of AD, and combination with acetylcholinesterase inhibitors should be explored.

\section{Statement of Ethics}

This research did not involve human participants or animals as this was a systematic review of existing publications and no primary data were collected. Written informed consent was, therefore, not obtained and ethical approval was not sought.

\section{Conflict of Interest Statement}

The authors have no conflicts of interest to declare.

\section{Funding Sources}

No funding sources to declare.

\section{Author Contributions}

The main systematic searches and the methodological studies were contributed by M.M.J. and A.Z. N.G.C. performed the additional systematic search. Writing, review, and editing were done by M.M.J. , J.A.G.A. and N.G.C. Conceptualization and supervision were performed by J.A.G.A. and N.G.C. All authors have read and approved the final version of the manuscript. 


\section{References}

1 Prince M, Knapp M, Guerchet M, McCrone P, Prina M, Comas-Herrera A, et al. Dementia UK: report to the Alzheimer's society. London: Alzheimer's Society; 2014. Available from: https://www.alzheimers.org.uk/sites/ default/files/migrate/downloads/dementia uk_update.pdf.

2 Fiest KM, Roberts JI, Maxwell CJ, Hogan DB, Smith EE, Frolkis A, et al. The prevalence and incidence of dementia due to Alzheimer's disease: a systematic review and meta-analysis. Can J Neurol Sci. 2016;43(Suppl 1):S51-82.

3 McKhann GM, Knopman DS, Chertkow H, Hyman BT, Jack CR Jr, Kawas CH, et al. The diagnosis of dementia due to Alzheimer's disease: recommendations from the National Institute on Aging-Alzheimer's Association workgroups on diagnostic guidelines for Alzheimer's disease. Alzheimers Dement. 2011; 7(3):263-9.

4 Michaud TL, Su D, Siahpush M, Murman DL. The risk of incident mild cognitive impairment and progression to dementia considering mild cognitive impairment subtypes. Dement Geriatr Cogn Dis Extra. 2017;7(1):15-29.

5 Mendonça MD, Alves L, Bugalho P. From subjective cognitive complaints to dementia. Am J Alzheimers Dis Other Demen. 2016;31: 105-14.

6 Jack CR Jr, Bennett DA, Blennow K, Carrillo MC, Dunn B, Haeberlein SB, et al. NIA-AA Research framework: toward a biological definition of Alzheimer's disease. Alzheimers Dement. 2018;14(4):535-62.

7 Knight R, Khondoker M, Magill N, Stewart R, Landau S. A systematic review and meta-analysis of the effectiveness of acetylcholinesterase inhibitors and memantine in treating the cognitive symptoms of dementia. Dement Geriatr Cogn Disord. 2018;45(3-4):131-51.

8 Kametani F, Hasegawa M. Reconsideration of amyloid hypothesis and tau hypothesis in Alzheimer's disease. Front Neurosci. 2018;12 25.

9 Arnold SE, Arvanitakis Z, Macauley-Rambach SL, Koenig AM, Wang HY, Ahima RS, et al. Brain insulin resistance in type 2 diabetes and Alzheimer disease: concepts and conundrums. Nat Rev Neurol. 2018;14(3):168-81.

10 García-Casares N, Jorge RE, García-Arnés JA, Acion L, Berthier ML, Gonzalez-Alegre P, et al. Cognitive dysfunctions in middle-aged type 2 diabetic patients and neuroimaging correlations: a cross-sectional study. J Alzheimers Dis. 2014;42(4):1337-46.

11 Cheng D, Noble J, Tang MX, Schupf N, Mayeux R, Luchsinger JA. Type 2 diabetes and late-onset Alzheimer's disease. Dement Geriatr Cogn Disord. 2011;31(6):424-30.

12 Cukierman-Yaffe T, Gerstein HC, Colhoun HM, Diaz R, García-Pérez L-E, Lakshmanan $\mathrm{M}$, et al. Effect of dulaglutide on cognitive impairment in type 2 diabetes: an exploratory analysis of the REWIND trial. Lancet Neurol. 2020;19(7):582-90.
13 García-Casares N, García-Arnés JA, GómezHuelgas R, Valdivielso-Felices P, GarcíaArias C, González-Santos P. Glucagon-like peptide-1 (GLP-1) mimetics: a new treatment for Alzheimer's disease. Rev Neurol. 2014 Dec 1;59(11):517-24.

14 Sebastião I, Candeias E, Santos MS, de Oliveira CR, Moreira PI, Duarte AI. Insulin as a bridge between type 2 diabetes and Alzheimer disease-how anti-diabetics could be a solution for dementia. Front Endocrinol. 2014;5:110.

15 Butterfield DA, Halliwell B. Oxidative stress, dysfunctional glucose metabolism and Alzheimer disease. Nat Rev Neurosci. 2019;20(3): 148-60.

16 Tzimopoulou S, Cunningham VJ, Nichols TE, Searle G, Bird NP, Mistry P, et al. A multicenter randomized proof-of-concept clinical trial applying $\left[{ }^{18} \mathrm{~F}\right]$ FDG-PET for evaluation of metabolic therapy with rosiglitazone $\mathrm{XR}$ in mild to moderate Alzheimer's disease. J Alzheimers Dis. 2010;22(4):1241-56.

17 Gejl M, Brock B, Egefjord L, Vang K, Rungby $\mathrm{J}$, Gjedde A. Blood-brain glucose transfer in Alzheimer's disease: effect of GLP-1 analog treatment. Sci Rep. 2017;7(1):17490.

18 Sato N, Morishita R. The roles of lipid and glucose metabolism in modulation of $\beta$-amyloid, tau, and neurodegeneration in the pathogenesis of Alzheimer disease. Front Aging Neurosci. 2015;7:199.

19 Yarchoan M, Arnold SE. Repurposing diabetes drugs for brain insulin resistance in Alzheimer disease. Diabetes. 2014;63(7):225361.

20 Liberati A, Altman DG, Tetzlaff J, Mulrow C, Gøtzsche PC, Ioannidis JP, et al. The PRISMA statement for reporting systematic reviews and meta-analyses of studies that evaluate health care interventions: explanation and elaboration. PLoS Med. 2009;6(7):e1000100.

21 Gejl M, Brock B, Egefjord L, Rungby J, Gjedde A. GLP-1 analog raises glucose transport capacity of blood brain barrier in Alzheimer's disease. J Cereb Blood Flow Metab. 2017;37: 21-2.

22 Gejl M, Brock B, Egefjord L, Vang K, Gjedde A, Rungby J. GLP-1 receptor agonism preserves parahippocampal glucose metabolism in Alzheimer's disease. Diabetologia. 2017;60: S81.

23 Gejl M, Egejord L, Møller A, Hansen SB, Vang $\mathrm{K}$, Rodell A, et al. GLP-1 analogue liraglutide prevents decline of brain glucose metabolism in Alzheimer's disease: randomized, placebocontrolled double-blinded clinical trial. Eur J Nucl Med Mol Imaging. 2015;42:S132-3.

24 Hamidovic A. Position on zinc delivery to olfactory nerves in intranasal insulin phase I-III clinical trials. Contemp Clin Trials. 2015; 45(Pt B):277-80.

25 Abbatecola AM, Lattanzio F, Molinari AM, Cioffi M, Mansi L, Rambaldi P, et al. Rosiglitazone and cognitive stability in older individuals with type 2 diabetes and mild cogni- tive impairment. Diabetes Care. 2010;33(8): 1706-11.

26 Claxton A, Baker LD, Wilkinson CW, Trittschuh EH, Chapman D, Watson GS, et al. Sex and apoE genotype differences in treatment response to two doses of intranasal insulin in adults with mild cognitive impairment or Alzheimer's disease. J Alzheimers Dis. 2013; 35(4):789-97.

27 Chen Y, Wang J, Wang LJ, Lin H, Huang PJ. Effect of different blood glucose intervention plans on elderly people with type 2 diabetes mellitus combined with dementia. Eur Rev Med Pharmacol Sci. 2017;21(11):2702-7.

28 Watson GS, Baker LD, Cholerton BA, Rhoads KW, Merriam GR, Schellenberg GD, et al. Effects of insulin and octreotide on memory and growth hormone in Alzheimer's disease. J Alzheimers Dis. 2009;18(3):595-602.

29 Claxton A, Baker LD, Hanson A, Trittschuh EH, Cholerton B, Morgan A, et al. Long-acting intranasal insulin detemir improves cognition for adults with mild cognitive impairment or early-stage Alzheimer's disease dementia. J Alzheimers Dis. 2015;44(3): 897-906.

30 Stein MS, Scherer SC, Ladd KS, Harrison LC. A randomized controlled trial of high-dose vitamin D2 followed by intranasal insulin in Alzheimer's disease. J Alzheimers Dis. 2011; 26(3):477-84.

31 Rosenbloom MH, Barclay TR, Pyle M, Owens $\mathrm{BL}$, Cagan AB, Anderson CP, et al. A singledose pilot trial of intranasal rapid-acting insulin in apolipoprotein E4 carriers with mildmoderate Alzheimer's disease. CNS Drugs. 2014;28(12):1185-9.

32 Reger MA, Watson GS, Green PS, Wilkinson CW, Baker LD, Cholerton B, et al. Intranasal insulin improves cognition and modulates beta-amyloid in early AD. Neurology. 2008; 70(6):440-8.

33 Reger MA, Watson GS, Green PS, Baker LD, Cholerton B, Fishel MA, et al. Intranasal insulin administration dose-dependently modulates verbal memory and plasma amyloid-beta in memory-impaired older adults. J Alzheimers Dis. 2008;13(3):323-31.

34 Reger MA, Watson GS, Frey WH, Baker LD, Cholerton B, Keeling ML, et al. Effects of intranasal insulin on cognition in memory-impaired older adults: modulation by APOE genotype. Neurobiol Aging. 2006;27(3):4518 .

35 Craft S, Claxton A, Baker LD, Hanson AJ Cholerton B, Trittschuh EH, et al. Effects of regular and long-acting insulin on cognition and Alzheimer's disease biomarkers: a pilot clinical trial. J Alzheimers Dis. 2017;57(4): 1325-34.

36 Craft S, Baker LD, Montine TJ, Minoshima S, Watson GS, Claxton A, et al. Intranasal insulin therapy for Alzheimer disease and amnestic mild cognitive impairment: a pilot clinical trial. Arch Neurol. 2012;69(1):29-38. 
37 Craft S, Raman R, Chow TW, Rafii MS, Sun CK, Rissman RA, et al. Safety, efficacy, and feasibility of intranasal insulin for the treatment of mild cognitive impairment and $\mathrm{Alz}$ heimer disease dementia: a randomized clinical trial. JAMA Neurol. 2020 Jun 22;77(9):111.

38 Sato T, Hanyu H, Hirao K, Kanetaka H, Sakurai H, Iwamoto T. Efficacy of PPAR- $\gamma$ agonist pioglitazone in mild Alzheimer disease. Neurobiol Aging. 2011;32(9):1626-33.

39 Risner ME, Saunders AM, Altman JF, Ormandy GC, Craft S, Foley IM, et al. Efficacy of rosiglitazone in a genetically defined population with mild-to-moderate Alzheimer's disease. Pharmacogenomics J. 2006;6(4):246-54.

40 Hildreth KL, Van Pelt RE, Moreau KL, Grigsby J, Hoth KF, Pelak V, et al. Effects of pioglitazone or exercise in older adults with mild cognitive impairment and insulin resistance: a pilot study. Dement Geriatr Cogn Dis Extra. 2015;5(1):51-63.

41 Harrington C, Sawchak S, Chiang C, Davies J, Donovan C, Saunders AM, et al. Rosiglitazone does not improve cognition or global function when used as adjunctive therapy to AChE inhibitors in mild-to-moderate $\mathrm{Alz}$ heimer's disease: two phase 3 studies. Curr Alzheimer Res. 2011;8(5):592-606.

42 Hanyu H, Sato T, Sakurai H, Iwamoto T. The role of tumor necrosis factor-alpha in cognitive improvement after peroxisome proliferator-activator receptor gamma agonist pioglitazone treatment in Alzheimer's disease. J Am Geriatr Soc. 2010;58(5):1000-1.

43 Hanyu H, Sato T, Kiuchi A, Sakurai H, Iwamoto T. Pioglitazone improved cognition in a pilot study on patients with Alzheimer's disease and mild cognitive impairment with diabetes mellitus. J Am Geriatr Soc. 2009;57(1): 177-9.

44 Gold M, Alderton C, Zvartau-Hind M, Egginton S, Saunders AM, Irizarry M, et al. Rosiglitazone monotherapy in mild-to-moderate Alzheimer's disease: results from a randomized, double-blind, placebo-controlled phase III study. Dement Geriatr Cogn Disord. 2010; 30(2):131-46.

45 Geldmacher DS, Fritsch T, McClendon MJ, Landreth G. A randomized pilot clinical trial of the safety of pioglitazone in treatment of patients with Alzheimer disease. Arch Neurol. 2011;68(1):45-50.

46 Watson GS, Cholerton BA, Reger MA, Baker LD, Plymate SR, Asthana S, et al. Preserved cognition in patients with early Alzheimer disease and amnestic mild cognitive impairment during treatment with rosiglitazone: a preliminary study. Am J Geriatr Psychiatry. 2005;13(11):950-8.
47 Koenig AM, Mechanic-Hamilton D, Xie SX, Combs MF, Cappola AR, Xie L, et al. Effects of the insulin sensitizer metformin in Alzheimer disease: pilot data from a randomized placebo-controlled crossover study. Alzheimer Dis Assoc Disord. 2017;31(2):107-13.

48 Luchsinger JA, Perez T, Chang H, Mehta P, Steffener J, Pradabhan G, et al. Metformin in amnestic mild cognitive impairment: results of a pilot randomized placebo controlled clinical trial. J Alzheimers Dis. 2016;51(2):50114.

49 Watson KT, Wroolie TE, Tong G, FolandRoss LC, Frangou S, Singh M, et al. Neural correlates of liraglutide effects in persons at risk for Alzheimer's disease. Behav Brain Res. 2019;356:271-8.

50 Gejl M, Gjedde A, Egefjord L, Møller A, Hansen $\mathrm{SB}$, Vang $\mathrm{K}$, et al. In Alzheimer's disease, 6-month treatment with GLP-1 analog prevents decline of brain glucose metabolism: randomized, placebo-controlled, doubleblind clinical trial. Front Aging Neurosci. 2016;8:108

51 Yildirim Simsir I, Soyaltin UE, Cetinkalp S. Glucagon like peptide-1 (GLP-1) likes Alzheimer's disease. Diabetes Metab Syndr. 2018; 12(3):469-75.

52 Mousa SA, Ayoub BM. Repositioning of dipeptidyl peptidase- 4 inhibitors and glucagon like peptide- 1 agonists as potential neuroprotective agents. Neural Regen Res. 2019;14(5): 745-8.

53 Khan MA, Alam Q, Haque A, Ashafaq M, Khan MJ, Ashraf GM, et al. Current progress on peroxisome proliferator-activated receptor gamma agonist as an emerging therapeutic approach for the treatment of Alzheimer's disease: an update. Curr Neuropharmacol. 2019;17(3):232-46.

54 Festuccia WT, Oztezcan S, Laplante M, Berthiaume M, Michel C, Dohgu S, et al. Peroxisome proliferator-activated receptor-gamma-mediated positive energy balance in the rat is associated with reduced sympathetic drive to adipose tissues and thyroid status. Endocrinology. 2008;149(5):2121-30.

55 Chang KL, Pee HN, Yang S, Ho PC. Influence of drug transporters and stereoselectivity on the brain penetration of pioglitazone as a potential medicine against Alzheimer's disease. Sci Rep. 2015;5:9000.

56 Craft S, Asthana S, Schellenberg G, Baker L, Cherrier M, Boyt AA, et al. Insulin effects on glucose metabolism, memory, and plasma amyloid precursor protein in Alzheimer's disease differ according to apolipoprotein-E genotype. Ann N Y Acad Sci. 2000;903:222-8.
57 Craft S, Asthana S, Schellenberg G, Cherrier $\mathrm{M}$, Baker LD, Newcomer J, et al. Insulin metabolism in Alzheimer's disease differs according to apolipoprotein E genotype and gender. Neuroendocrinology. 1999;70(2): 146-52.

58 Reiman EM, Chen K, Alexander GE, Caselli RJ, Bandy D, Osborne D, et al. Functional brain abnormalities in young adults at genetic risk for late-onset Alzheimer's dementia. Proc Natl Acad Sci U S A. 2004;101(1):284-9.

59 Valla J, Yaari R, Wolf AB, Kusne Y, Beach TG, Roher AE, et al. Reduced posterior cingulate mitochondrial activity in expired young adult carriers of the APOE $\varepsilon 4$ allele, the major lateonset Alzheimer's susceptibility gene. J Alzheimers Dis. 2010;22(1):307-13.

60 Benedict C, Hallschmid M, Schmitz K, Schultes B, Ratter F, Fehm HL, et al. Intranasal insulin improves memory in humans: superiority of insulin aspart. Neuropsychopharmacology. 2007;32(1):239-43.

61 Wallach JD, Wang K, Zhang AD, Cheng D, Grossetta Nardini HK, Lin H, et al. Updating insights into rosiglitazone and cardiovascular risk through shared data: individual patient and summary level meta-analyses. BMJ. 2020; 368:17078.

62 Cao B, Rosenblat JD, Brietzke E, Park C, Lee Y, Musial N, et al. Comparative efficacy and acceptability of antidiabetic agents for Alzheimer's disease and mild cognitive impairment: a systematic review and network metaanalysis. Diabetes Obes Metab. 2018;20(10): 2467-71.

63 Moon JH, Kim HJ, Yang AH, Kim HM, Lee BW, Kang ES, et al. The effect of rosiglitazone on LRP1 expression and amyloid $\beta$ uptake in human brain microvascular endothelial cells: a possible role of a low-dose thiazolidinedione for dementia treatment. Int J Neuropsychopharmacol. 2012;15:135-42.

64 Jojo GM, Kuppusamy G, De A, Karri VVSNR. Formulation and optimization of intranasal nanolipid carriers of pioglitazone for the repurposing in Alzheimer's disease using BoxBehnken design. Drug Dev Ind Pharm. 2019; 45(7):1061-72.

65 Iketani R, Ohno K, Kawasaki Y, Matsumoto K, Yamada H, Kishino S. Apolipoprotein E gene polymorphisms affect the efficacy of thiazolidinediones for Alzheimer's disease: a systematic review and meta-analysis. Biol Pharm Bull. 2018;41(7):1017-23.

66 Femminella GD, Frangou E, Love SB, Busza G, Holmes C, Ritchie C, et al. Evaluating the effects of the novel GLP-1 analogue liraglutide in Alzheimer's disease: study protocol for a randomised controlled trial (ELAD study). Trials. 2019;20(1):191. 